\title{
Efficacy of Vildagliptin Added to Continuous Subcutaneous Insulin Infusion (CSII) in Hospitalized Patients with Type 2 Diabetes
}

\author{
Fu-ping Lyu • Bing-kun Huang • Wei-juan Su • Fang-fang Yan • \\ Jin-yang Zeng · Zheng Chen · Yu-xian Zhang · Shun-hua Wang • \\ Yin-xiang Huang · Mu-lin Zhang · Xiu-lin Shi • Ming-zhu Lin • \\ Xue-jun Li
}

Received: October 10, 2019 / Published online: February 4, 2020

(C) The Author(s) 2020

\section{ABSTRACT}

Introduction: The aim of this study was to compare the efficacy of vildagliptin as add-on therapy to short-term continuous subcutaneous insulin infusion (CSII) with CSII monotherapy in hospitalized patients with type 2 diabetes mellitus (T2DM).

Methods: A total of 200 hospitalized patients with inadequately controlled T2DM were randomized into groups, with one group receiving CSII monotherapy (CSII group, $n=100$ ) and the

Enhanced Digital Features To view enhanced digital features for this article go to https://doi.org/10.6084/ m9.figshare.11499909.

F. Lyu - B. Huang - W. Su · F. Yan · J. Zeng

Z. Chen · Y. Zhang · S. Wang · X. Shi · M. Lin (ه) .

X. Li (ه)

Department of Endocrinology and Diabetes, The

First Affiliated Hospital of Xiamen University,

Xiamen, China

e-mail: linmz65@tom.com

X. Li

e-mail: xmlixuejun@163.com

F. Lyu - B. Huang · W. Su · F. Yan · J. Zeng

Z. Chen $\cdot$ Y. Zhang $\cdot$ S. Wang $\cdot$ Y. Huang ·

M. Zhang $\cdot$ X. Shi $\cdot$ M. Lin $\cdot$ X. Li

Xiamen Diabetes Institute, The First Affiliated

Hospital of Xiamen University, Xiamen, China

Y. Huang $\cdot$ M. Zhang $\cdot$ M. Lin $\cdot$ X. Li

Fujian Provincial Key Laboratory of Translational

Medicine for Diabetes, Xiamen, China other group receiving CSII plus vildagliptin as add-on (CSII + Vig group, $n=100)$. Of these, 191 completed the 7-day trial (CSII group, $n=99 ;$ CSII + Vig group, $n=92$ ) and included in the analysis. The glycemic control and variability of the patients were measured using allday capillary blood glucose (BG) monitoring. Weight and fasting C-peptide levels were evaluated before and after the interventions.

Results: Mean BG concentrations during the whole treatment period were lower and the time to reach target BG was reduced in the CSII + Vig group compared with the CSII group $(9.89 \pm 3.37$ vs. $9.46 \pm 3.23 \mathrm{mmol} / \mathrm{L}, P<0.01$; $129 \pm 4$ vs. $94 \pm 5 \mathrm{~h}, P<0.01$, respectively). Similarly, the indicators of glycemic variability, namely the standard deviation of BG and the largest amplitude of glycemic excursion, were significantly decreased in the CSII + Vig group compared with the CSII group $(2.68 \pm 1.05$ vs. $2.39 \pm 1.00 \mathrm{mmol} / \mathrm{L}, \quad P<0.01 ; 7.19 \pm 2.86$ vs. $6.23 \pm 2.73 \mathrm{mmol} / \mathrm{L}, P<0.01$, respectively).

Conclusions: Short-term CSII with vildagliptin as add-on therapy may be an optimized treatment for hospitalized patients with T2DM compared with short-term CSII monotherapy.

Keywords: Continuous subcutaneous insulin infusion; Dipeptidyl peptidase-4 inhibitors; Glycemic variability; Hypoglycemia 


\section{Key Summary Points}

Inpatients with inadequately controlled T2DM were randomized into two groups and treated for 7 days with CSII alone (CSII group) or with CSII plus vildagliptin in our present randomized trial.

The intervention in which vildagliptin was added to a CSII-based intensive treatment in patients with T2DM was effective in improving glucose levels, glycemic excursions and reduced incidence of hypoglycemia, target blood glucose quickly, and showed no weight gain compared with CSII alone.

Accordingly CSII plus vildagliptin appears to be a beneficial alternative regimen for the management of uncontrolled hyperglycemia in patients with type 2 diabetes.

\section{INTRODUCTION}

Type 2 diabetes mellitus (T2DM) is a chronic disease characterized by progressive $\beta$-cell dysfunction that leads to insulin deficiency. In China, primary healthcare coverage is insufficient to provide the extent of care required, with the result that a considerable number of patients with diabetes suffer from sustaining hyperglycemia, leading to hospitalization for treatment. These patients frequently receive intensive insulin therapies during their hospitalization, such as continuous subcutaneous insulin infusion (CSII) and multiple subcutaneous insulin injection. It has been suggested that short-term intensive insulin therapy can rapidly relieve glucose toxicity due to high glucose levels, ameliorate the state of insulin resistance and restore islet $\beta$-cell function in persons with poorly controlled T2DM [1, 2]. As a real-time regulation therapy, CSII is potentially the best and strongest intensive glucoselowering intervention currently available $[3,4]$. However, glycemic fluctuations and hypoglycemia are unavoidable risks associated with
CSII $[5,6]$. Results from a number of studies suggest that glycemic disturbances are also an important factor leading to chronic complications of diabetes [7-9], with some authors even suggesting that glycemic variability has more detrimental effects than persistent hyperglycemia in the progression of diabetic complications [10, 11]. Therefore, alternative treatment regimens are needed that could improve glycemic control while minimizing glycemic variability and the risk of hypoglycemia in patients with diabetes.

Dipeptidyl peptidase-4 (DPP-4) inhibitors can increase the plasma level of endogenous incretins by preventing their inactivation, thereby modifying insulin secretion from $\beta$-cells and glucagon release from $\alpha$-cells in a glucosedependent manner [12]. In one study, the DDP4 inhibitor vildagliptin as an add-on to insulin treatment for 24 months was well tolerated and led to sustained reductions in glycated hemoglobin (HbA1c), the dose and number of insulin injections and the risk of hypoglycemia [13]. However, reports on the efficacy of adding vildagliptin to short-term insulin therapy are rare. In the study reported here, vildagliptin was added to short-term CSII to control the glucose level of patients with T2DM inadequately controlled on CSII monotherapy to evaluate the impact of the combination therapy on glycemic variability.

The aim of this randomized controlled trial was to compare the effects of CSII monotherapy with those of CSII combined with vildagliptin in hospitalized T2DM patients, in an attempt to achieve a more effective and safer therapeutic regimen.

\section{METHODS}

\section{Subjects}

A total of 200 inpatients diagnosed with T2DM according to the 1999 World Health Organization diagnostic criteria were enrolled in the study and assessed for eligibility in the Department of Endocrinology and Diabetes, First Affiliated Hospital, Xiamen University, Xiamen, China from November 2018 to March 2019. The 
inclusion criteria were based on demographic factors and the results of preliminary tests, as follows: (1) age between 30 and 70 years, HbA1c level $\geq 8.5 \%$ and a body mass index (BMI) of between 18 and $28 \mathrm{~kg} / \mathrm{m}^{2}$; (2) negative for glutamic acid decarboxylase autoantibody, antiislet cell autoantibody and anti-insulin autoantibody; and (3) sustained treatment for 2 months without a known treatment history of DPP4-inhibitor or glucagon-like peptide-1 (GLP1) receptor agonist or without any anti-hyperglycemia drugs. The exclusion criteria were: (1) type 1 diabetes, gestational diabetes or diabetes with a secondary cause (such as taking medications known to affect glycemic control); (2) significant renal impairment (estimated creatinine clearance $<50 \mathrm{~mL} / \mathrm{min}$ ) or elevated alanine or aspartate aminotransferase levels or congestive heart failure (New York Heart Association Functional Classification III-IV); (3) occurrence of any severe diabetic complications or infection in the previous 3 months; and (4) scheduled surgery or serious trauma or any mental health condition. Other patients whom the investigators judged to be inappropriate for inclusion in the study were also excluded.

\section{Study Design and Intervention}

This study was a single-center, randomized controlled, open-label clinical trial. The random number table was computer generated. The eligible subjects were randomized using the random number table into treatment groups receiving either CSII alone (CSII group, $n=100$ ) or CSII combined with $50 \mathrm{mg}$ Galvus vildagliptin (Novartis, Basel, Switzerland) twice daily (CSII + Vig group, $n=100)$ for 7 days. During the 7-day treatment period, one patient in the CSII group and eight patients in the CSII + Vig group discontinued due to protocol violation or personal request to withdraw. The remaining patients, all treated with Humalog rapid-acting insulin (insulin lispro; Eli Lilly, Indianapolis, IN, USA) using the MiniMed Paradigm ${ }^{\circledR} 722$ insulin pump (Medtronic, Northridge, CA, USA), completed the trial and their data were used in the analysis (see Fig. 1). The initial daily insulin dosage was calculated as follows: total

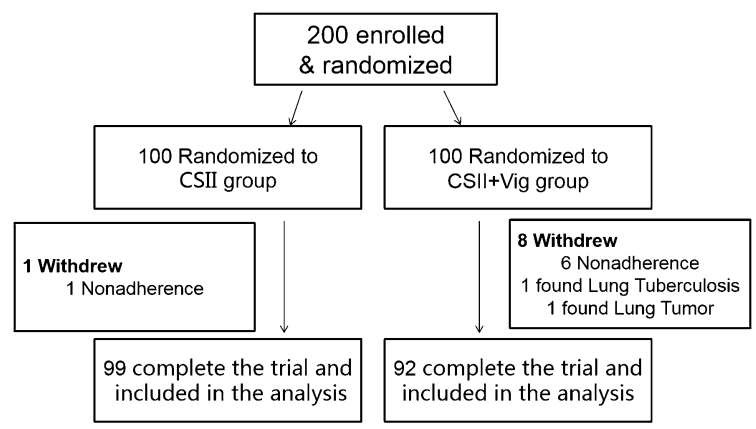

Fig. 1 Flowchart of patient inclusion throughout the trial. CSII continuous subcutaneous insulin infusion monotherapy group, CSII+Vig group CSII therapy plus vildagliptin as add-on group

insulin dose daily $=0.7$ unit $\times$ body weight (kg). The basal rate (units/h) was calculated as $50 \%$ of the total insulin dose, and the other $50 \%$ was administered as a preprandial bolus before each meal. The dawn phenomenon and nocturnal hypoglycemia were taken into account, and the basal rate was fixed depending on the time period: basal insulin dose $/ 24 \times 0.8$ between 2200 and 0300 hours; basal insulin dose $/ 24 \times 1.2$ between 0300 and 0700 hours; basal insulin dose $/ 24 \times 1.0$ between 0700 and 2200 hours. Capillary blood glucose (BG) was monitored 7 times per day (before and $2 \mathrm{~h}$ after each meal and at bedtime) by a trained nurse using a unified glucometer (Johnson \& Johnson, New Brunswick, NJ, USA). Incidences of hypoglycemia were detected by sampling capillary BG $(<3.9 \mathrm{mmol} / \mathrm{L})$ when patients suffered from hypoglycemic-like symptoms or in samples taken for regular measurements.

The basal and bolus doses of insulin infusion were tailored every 2 days by one doctor by 0.3 unit/h and 3 units, respectively, according to the capillary BG level to achieve the glycemic target (fasting $\mathrm{BG}<8.0 \mathrm{mmol} / \mathrm{L}$ and average postprandial $\mathrm{BG}<10.0 \mathrm{mmol} / \mathrm{L}$ ). All of the patients underwent the same education program on lifestyle management, including diet and exercise counseling. A diabetic diet consisting of $50 \%$ carbohydrate $(200 \mathrm{~g}), 35 \%$ fat and $15 \%$ protein was provided for all patients during the intervention. Regular physical exercise, such as walking, jogging or climbing stairs, was recommended after meals for 30 minutes. 
This work was approved by the Medical Research and Ethics Committee of the First Affiliated Hospital, Xiamen University (Xiamen, China). This study was registered on the International Clinical Trials Registry Platform (ICTRP) with trial registration identifier number NCT03563794. Informed consent was obtained from all participants in this study.

\section{Measurements}

Anthropometric and laboratory data, including height, weight, age, BMI, fasting plasma glucose (FPG), HbA1c, fasting C-peptide (FC-P), triglycerides (Tg), total cholesterol (TC), high-density lipoprotein-cholesterol (HDL-C) and low-density lipoprotein-cholesterol (LDL-C), were obtained for all patients before CSII treatment. Weight, FPG and FC-P levels were re-examined after the interventions.

The mean BG level (MBG), standard deviation of BG levels (SDBG), largest amplitude of glycemic excursions (LAGE) and proportion (\%) of glucose concentration were calculated by allday capillary BG monitoring. The change in body weight and FC-P before and after treatment were calculated, and the number of hours to achieve the glycemic target was recorded based on daily glucose monitoring.

\section{Statistical Methods}

All statistical analyses were performed using SPSS version 18.0 software (IBM Corp, Armonk, NY, USA). The variables were then examined independently and subjected to normality and homogeneity of variance tests. The data for normally distributed variables are reported as the mean \pm standard deviation (SD), and the data for nonnormally distributed variables are reported as medians and interquartile ranges. The independent-samples $t$ test was used to test for differences between the two treatment groups. Count data are expressed as rates and compared using Chi-square analysis. A twosided value of $P<0.05$ was considered to indicate statistical significance.

\section{RESULTS}

\section{Baseline Characteristics}

Of the 200 inpatients with uncontrolled T2DM who were enrolled in the study and randomized into the two treatment groups (CSII group and CSII + Vig group), 191 completed the 7-day trial and were included in the analysis (see Fig. 1). No serious adverse effects were observed during the intervention in both groups. The baseline features and clinical characteristics of the patients (age, gender, weight and BMI) were similar in the two groups $(P>0.05$; Table 1$)$. There were also no significant differences between the groups in terms of glucose levels (HbA1c, glycated albumin [GA], FPG) and lipid profile (TC, HDL-C, LDL-C, and Tg) at the beginning of the study $(P>0.05$; Table 1$)$. The constituent ratio of different diabetic complications and treatment methods before trial were also not significantly different between the groups $(P>0.05$, Table 1$)$.

\section{Treatment Efficacy of Glycemic Control}

The indices of glycemic control derived from the capillary BG monitoring during the 7 days of treatment are shown in Table 2. Patients in both groups achieved prompt and sustained improvement in overall MBG level during the 7 days of treatment. The overall MBG levels $( \pm$ SD) were significantly lower in the CSII + Vig group than in the CSII group $(9.89 \pm 3.37$ vs. $9.46 \pm 3.23 \mathrm{mmol} / \mathrm{L}, P<0.01$; Table 2). Also, the comparison of daily MBG levels showed that there was a significant difference between the CSII and CSII + Vig groups on days 3 to 7 of treatment (Fig. 2). During treatment, the proportion of glucose readings between 3.9 and 7.8 $\mathrm{mmol} / \mathrm{L}$ (P7.8, \%) and between 3.9 and $10.0 \mathrm{mmol} / \mathrm{L} \quad(\mathrm{P} 10.0, \%)$ was significantly higher in the CSII + Vig group than in the CSII group $(36.14$ vs. $31.52 \%[P<0.01]$ and 62.96 vs. $57.10 \%[P<0.01]$, respectively; Table 2$)$. The incidence of patients who ultimately achieved the glycemic target (fasting $\mathrm{BG}<8.0 \mathrm{mmol} / \mathrm{L}$ and average postprandial $\mathrm{BG}<10.0 \mathrm{mmol} / \mathrm{L}$ ) in the CSII + Vig group was $79.3 \%$ (73/92), which 
Table 1 Baseline comparisons between the continuous subcutaneous insulin infusion (CSII) alone group and the CSII plus vildagliptin as add-on group before treatment

\begin{tabular}{|c|c|c|c|}
\hline \multirow[t]{2}{*}{ Characteristic } & \multicolumn{2}{|c|}{ Treatment group } & \multirow[t]{2}{*}{$P$ value } \\
\hline & $\overline{\text { CSII }}$ & CSII + Vig & \\
\hline Patients, $n$ & 99 & 92 & - \\
\hline Gender, $n$ (male/female) & $65 / 34$ & $49 / 43$ & 0.08 \\
\hline Mean age, years & $50.94 \pm 11.58$ & $49.46 \pm 11.64$ & 0.38 \\
\hline$<60(n)$ & $70.7 \%(70 / 99)$ & $79.3 \%(73 / 92)$ & 0.17 \\
\hline$\geq 60(n)$ & $29.3 \%(29 / 99)$ & $20.7 \%(19 / 92)$ & \\
\hline Duration diabetes, years & $3 \pm 0.83$ & $4 \pm 0.17$ & 0.95 \\
\hline Weight, kg & $69.02 \pm 14.00$ & $66.58 \pm 12.73$ & 0.21 \\
\hline BMI, $\mathrm{kg} / \mathrm{m}^{2}$ & $24.80 \pm 3.14$ & $24.34 \pm 3.19$ & 0.32 \\
\hline HbAlc, \% & $10.94 \pm 2.07$ & $11.03 \pm 1.87$ & 0.77 \\
\hline$<9 \%(n)$ & $17.2 \%(17 / 99)$ & $12.0 \%(11 / 92)$ & 0.31 \\
\hline$\geq 9 \%(n)$ & $82.8 \%(82 / 99)$ & $88.0 \%(81 / 92)$ & \\
\hline Admission random $\mathrm{BG}, \mathrm{mmol} / \mathrm{L}$ & $14.37 \pm 4.65$ & $13.67 \pm 5.18$ & 0.33 \\
\hline Admission fasting $\mathrm{BG}, \mathrm{mmol} / \mathrm{L}$ & $8.34 \pm 2.84$ & $8.74 \pm 2.85$ & 0.34 \\
\hline FC-P, ng/mL & $1.36 \pm 1.05$ & $1.29 \pm 0.91$ & 0.69 \\
\hline Systolic blood pressure, $\mathrm{mmHg}$ & $131.93 \pm 17.32$ & $130.46 \pm 15.60$ & 0.53 \\
\hline Diastolic blood pressure, $\mathrm{mmHg}$ & $81.82 \pm 10.19$ & $81.51 \pm 7.65$ & 0.81 \\
\hline Alanine aminotransferase, units/L & $28.34 \pm 19.40$ & $28.06 \pm 24.35$ & 0.93 \\
\hline Aspartate aminotransferase, units/L & $22.54 \pm 12.96$ & $20.80 \pm 9.74$ & 0.32 \\
\hline Cre, $\mathrm{mmol} / \mathrm{L}$ & $61.18 \pm 16.39$ & $56.78 \pm 14.47$ & 0.07 \\
\hline $\mathrm{TC}, \mathrm{mmol} / \mathrm{L}$ & $5.11 \pm 1.24$ & $5.36 \pm 1.07$ & 0.15 \\
\hline $\mathrm{HDL}-\mathrm{C}, \mathrm{mmol} / \mathrm{L}$ & $1.21 \pm 0.31$ & $1.28 \pm 0.49$ & 0.29 \\
\hline $\mathrm{LDL}-\mathrm{C}, \mathrm{mmol} / \mathrm{L}$ & $2.84 \pm 0.87$ & $3.01 \pm 0.93$ & 0.26 \\
\hline $\mathrm{Tg}, \mathrm{mmol} / \mathrm{L}$ & $2.03 \pm 1.93$ & $2.06 \pm 1.84$ & 0.90 \\
\hline \multicolumn{4}{|l|}{ Diabetic complications } \\
\hline Peripheral vascular disease & $27.3 \%(27 / 99)$ & $22.8 \%(21 / 92)$ & 0.51 \\
\hline Retinopathy $(n)$ & $50.5 \%(50 / 99)$ & $52.2 \%(48 / 92)$ & 0.77 \\
\hline Neuropathy $(n)$ & $25.3 \%(25 / 99)$ & $21.7 \%(20 / 92)$ & 0.70 \\
\hline Nephropathy $(n)$ & $18.2 \%(18 / 99)$ & $15.2 \%(14 / 92)$ & 0.57 \\
\hline \multicolumn{4}{|l|}{ Treatment before CSII } \\
\hline Diet alone $(n)$ & $38.3 \%(38 / 99)$ & $36.4 \%(36 / 92)$ & 0.91 \\
\hline Oral drugs $(n)$ & $38.3 \%(38 / 99)$ & $31.5 \%(29 / 92)$ & 0.32 \\
\hline
\end{tabular}


Table 1 continued

\begin{tabular}{llll}
\hline Characteristic & Treatment group & \multirow{2}{*}{ value } \\
\cline { 2 - 3 } & CSII & CSII + Vig & \\
\hline Insulin alone $(n)$ & $11.1 \%(11 / 99)$ & $13.0 \%(12 / 92)$ & 0.68 \\
Insulin plus oral drugs $(n)$ & $12.1 \%(12 / 99)$ & $16.3 \%(15 / 92)$ & 0.41 \\
\hline
\end{tabular}

Data in table are presented as the mean \pm standard deviation (SD) or medians (interquartile range), as a number $(n)$ or as a percentage with the ratio of patients given in parenthesis

CSII group Continuous subcutaneous insulin infusionI monotherapy group, CSII + Vig group CSII therapy in combination with vildagliptin as add-on group, $B M I$ body mass index, $H b A 1 c$ glycated hemoglobin, $B G$ blood glucose, $F C-P$ fasting C-peptide, $C r e$ creatinine, $T C$ total cholesterol, $T g$ triglycerides, $H D L-C$ high-density lipoprotein-cholesterol, $L D L-C$ lowdensity lipoprotein-cholesterol

Table 2 Glycemic control and insulin dose between the continuous subcutaneous insulin infusion (CSII) alone group and the CSII plus vildagliptin as add-on group

\begin{tabular}{llll}
\hline Characteristics & CSII $(n)$ & CSII + Vig $(n)$ & $P$ value \\
\hline Total number of BG tests given & 4448 & 4009 & - \\
Overall AUC of BG, mmol/l h & $1512 \pm 22$ & $1453 \pm 25$ & 0.09 \\
Mean overall BG concentration, mmol/L & $9.89 \pm 3.37$ & $9.46 \pm 3.23$ & $<0.01$ \\
Percentage of BG readings between 3.9 and $7.8 \mathrm{mmol} / \mathrm{L}(n)$ & $31.52 \%(1402 / 4448)$ & $36.14 \%(1449 / 4009)$ & $<0.01$ \\
Percentage of BG readings between 3.9 and $10.0 \mathrm{mmol} / \mathrm{L}(n)$ & $57.10 \%(2540 / 4448)$ & $62.96 \%(2524 / 4009)$ & $<0.01$ \\
Percentage of BG readings $>10.0 \mathrm{mmol} / \mathrm{L}(n)$ & $42.9 \%(1907 / 4448)$ & $37.04 \%(1485 / 4009)$ & $<0.01$ \\
Percentage of BG readings $<3.9 \mathrm{mmol} / \mathrm{L}(n)$ & $4.04 \%(18 / 4448)$ & $1.75 \%(7 / 4009)$ & 0.05 \\
Percentage of patients achieving euglycemia $(n)$ & $67.7 \%(67 / 99)$ & $79.3 \%(73 / 92)$ & $<0.01$ \\
Time to achieve euglycemia, h & $129 \pm 4$ & $94 \pm 5$ & $<0.01$ \\
Mean SDBG, mmol/L & $2.68 \pm 1.05$ & $2.39 \pm 1.00$ & $<0.01$ \\
Mean LAGE, mmol/L & $7.19 \pm 2.86$ & $6.23 \pm 2.73$ & $<0.01$ \\
Total insulin dose, units per day per kg & $0.74 \pm 0.39$ & $0.69 \pm 0.17$ & 0.27 \\
$\Delta$ Weight, kg & $-0.43 \pm 0.23$ & $-0.50 \pm 0.25$ & 0.85 \\
$\Delta$ FC-P, ng/mL & $+0.26 \pm 0.99$ & $+0.18 \pm 0.78$ & 0.60 \\
\hline
\end{tabular}

Data in table are presented as the mean $\pm S D$ or as a percentage with the ratio of patients given in parenthesis $A U C$ Area under the time-concentration curve, $S D B G$ standard deviation of blood glucose, $L A G E$ largest amplitude of glycemic excursion

was higher than that observed in the CSII group (67.7\%; 67/99). In addition, the time to reach target BG level was lower in the CSII + Vig group than in the CSII group (94 \pm 5 vs. $129 \pm 4$ h, $P<0.01$; Table 2).

\section{Comparison of Glycemic Variability}

The mean SDBG and LAGE of the patients in the CSII + Vig group during 7 days of treatment were both significantly lower than those of the CSII group after the intervention (both 


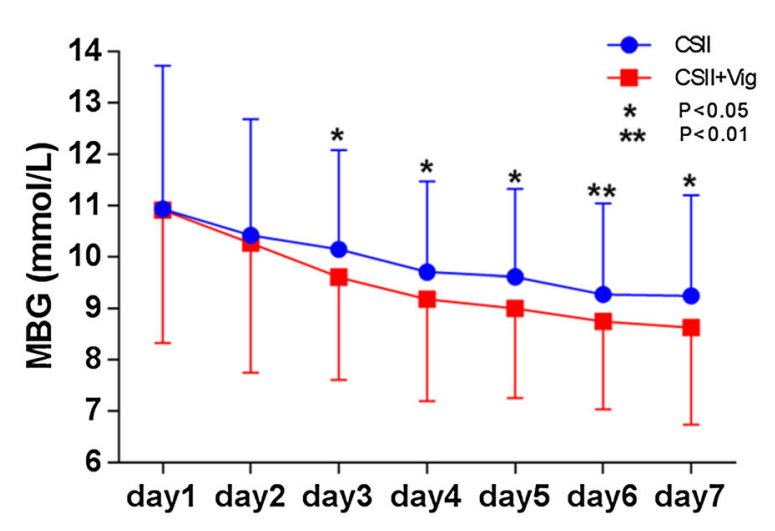

Fig. 2 Comparison of the daily changes in mean blood glucose $(M B G)$ level between the CSII and CSII+Vig groups. Data are presented as the mean \pm standard deviation (SD). MBG refers to the MBG concentration of each day during treatment

$P<0.01$; Table 2). After 1 week of treatment, the proportion of $B G$ readings which indicated hypoglycemia $(\mathrm{BG}<3.9 \mathrm{mmol} / \mathrm{L})$ in the CSII + Vig group was $1.75 \%$ (7/4009 measurements), which was lower than that observed in the CSII group (4.04\%, 18/4448 measurements). It is noteworthy that the CSII + Vig group achieved better improvements in MBG concentrations after lunch and after dinner (Fig. 3).

\section{Effects on Insulin Dose and Weight}

Over the 7-day trial period, the standardized insulin dose $( \pm$ SD) used to achieve target glucose in the CSII + Vig group and CSII group was $0.69 \pm 0.17$ and $0.74 \pm 0.39$ units/day $/ \mathrm{kg}$, respectively; the difference was not significant. The weight change and FC-P change from baseline in the two groups were also not significant $(P>0.05$; Table 2$)$.

\section{DISCUSSION}

In this study we assessed the clinical efficacy of vildagliptin in patients with inadequately controlled T2DM receiving CSII treatment, including indicators such as reduction in glucose excursion and amelioration of glucose fluctuation. Although an improvement in glycemic control using the combination of a DPP-4 inhibitor and insulin has been demonstrated in previous studies, the intervention time in those studies was between 24 weeks and 2 years [13-17]. Our results show that the MBG level and the BG level after meals were ameliorated when vildagliptin was added to CSII monotherapy during our short-term treatment period of 7 days, with a significant difference in the MBG manifesting from the third day onwards. It is known that DDP-4 inhibitors can inhibit the degradation of the endogenous incretin hormones GLP-1 and gastric inhibitory polypeptide (GIP), which in turn promotes insulin secretion and inhibits glucagon secretion in a glucose-dependent manner, thus helping to correct hyperglycemic states [18]. In one study, patients receiving CSII therapy with vildagliptin as add-on achieved restoration of islet $\beta$-cell function more effectively than those receiving CSII monotherapy, which resulted in a greater improvement in glycemic control [2].

Glucose variability, characterized by extreme glucose excursions, has been found to have the potential to be a predictor of diabetic complications, independent of HbA1c levels in patients with T2DM [19]. Glucose variability has also been shown to increase the risk of diabetic complications, mainly due to the activation of oxidative stress and the innate immune system [20]. Results from earlier studies suggest that add-on vildagliptin therapy is significantly well tolerated and improves HbA1c, fasting BG and postprandial BG levels in persons with T2DM compared with placebo [14-17]. Our results also suggest that the risk of hypoglycemia may be reduced when vildagliptin is added to insulin therapy: the frequency of readings indicating hypoglycemia was decreased in the CSII + Vig group. This improvement may be attributed to the ability of vildagliptin to block GLP-1 and GIP inactivation by DPP-4, thereby improving glucagon dynamics during hypoglycemia and food rechallenge [21].

The problems of weight and economics need to be considered in the management of T2DM patients hospitalized due to hyperglycemia [22]. Vildagliptin has been shown to be effective and well tolerated in various treatment regimens, with a neutral effect on body weight, possibly 


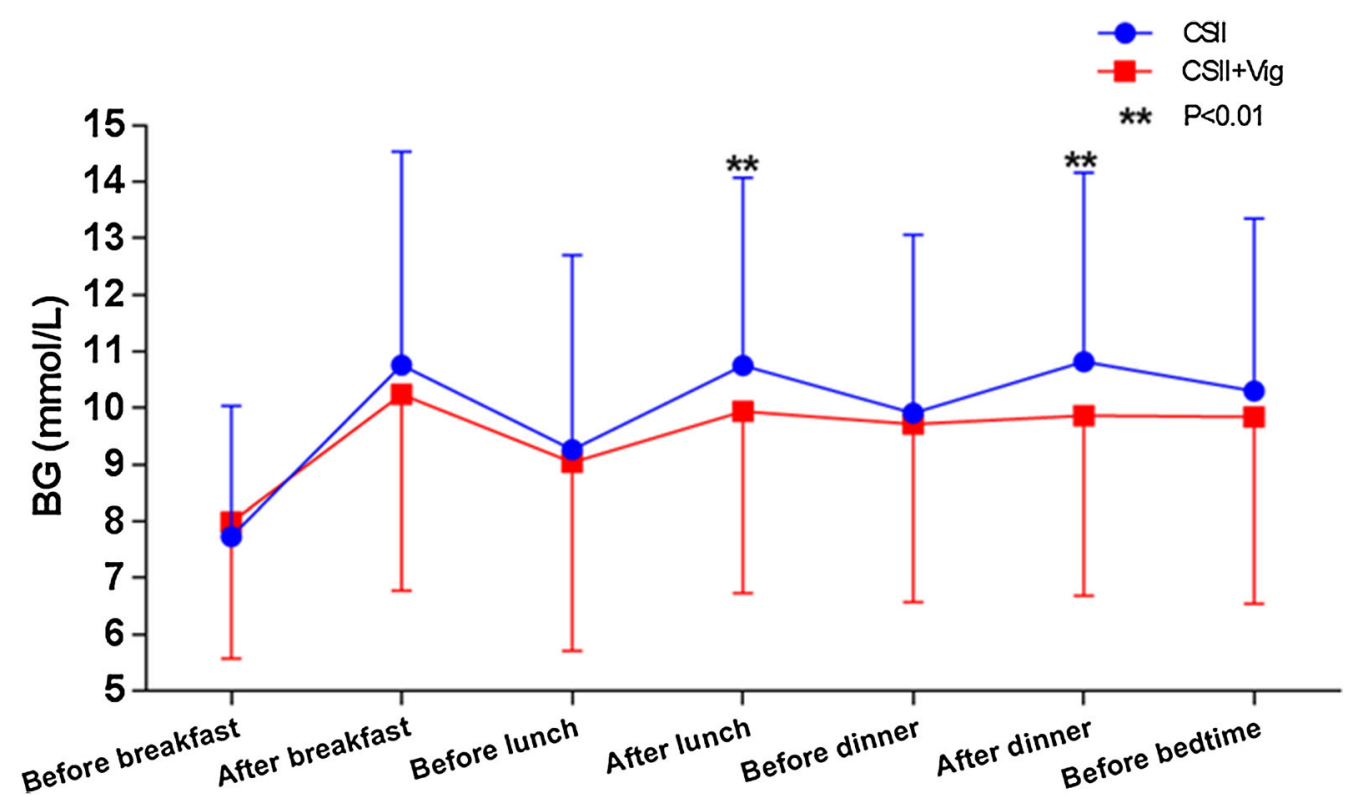

Fig. 3 Overall changes in blood glucose (BG) during the daytime between the CSII and CSII+Vig groups. Data are presented as the mean $\pm \mathrm{SD}$. BG refers to the $\mathrm{MBG}$ concentration of each regular measurement

because pharmacologic action augments the GLP-1 effect [13, 23]. Additionally, previous studies have indicated that vildagliptin as addon therapy to various insulin regimens (but not including CSII) can decrease daily insulin doses and improve glycemic control without weight gain [24-26]. However, in our study, we observed no significant decrease in insulin dose after augmenting CSII with vildagliptin, likely due to the short intervention time and small sample size, although improved glycemic control was achieved without weight gain or an increase in the incidence of hypoglycemia. More importantly, in comparison to CSII monotherapy, a significantly short time was required to achieve the glycemic goal when vildagliptin was added to CSII, which suggests that this combined therapeutic regimen may shorten hospital stays and reduce hospitalization expenses for patients. We therefore suggest that vildagliptin as add-on therapy to CSII can be a cost-effective strategy to achieve the glycemic target.

There are several limitations to our study. First, it was a short-term study and, as such, the intervention time was too short to determine changes in better indicators of glucose excursion, such as HbA1c, GA, and other longterm trends in biochemical indicators. Complications may not have become obvious due to the short duration of the trial. Second, we initially planned to enroll 400 patients, but ultimately had to accept and randomize only 200 patients after exclusion. One major reason for this was that patients were screened more slowly than expected, with the result that the target enrollment for the trial was not reached by the end of the enrollment phase. The statistical calculation was completed at the end of the enrollment phase, and as the statistical results showed differences, the sample size was not further expanded. Third, in our study, glucose variation was calculated by measuring capillary BG glucose level instead of using the continuous glucose monitoring system; this was a limitation threshold because intermittent glucose monitoring only allows part of the whole picture to be detected. Finally, the incidence of hypoglycemia was based on glucose readings that were determined on capillary blood samples tested periodically or reported according to the patient's symptoms; in both cases, it is likely that asymptomatic hypoglycemia events were missed. 


\section{CONCLUSIONS}

In summary, the results of our randomized trial show that compared to CSII monotherapy, vildagliptin added onto a CSII-based intensive treatment was effective in improving glucose levels and glycemic excursions, reducing the incidence of hypoglycemia events and achieving the target BG level quickly in patients with T2DM, with the additional advantage of the patients showing no weight gain. We suggest that vildagliptin as add-on to CSII therapy might be a good option when the aim is to relieve glucotoxicity within a short period of hospitalization given that CSII during hospitalization is one of the most rapid and cost-effective therapies but is associated with several disadvantages, such as the risk of hypoglycemia.

\section{ACKNOWLEDGEMENTS}

Funding. No funding or sponsorship was received for this study or publication of this article. The Rapid Service Fee was funded by the authors.

Authorship. All named authors meet the International Committee of Medical Journal Editors (ICMJE) criteria for authorship for this article, take responsibility for the integrity of the work as a whole, and have given their approval for this version to be published.

Disclosures. Fu-ping Lyu, Bing-kun Huang, Wei-juan Su, Fang-fang Yan, Jin-yang Zeng, Zheng Chen, Yu-xian Zhang, Shun-hua Wang, Yin-xiang Huang, Mu-lin Zhang, Xiu-lin Shi, Ming-zhu Lin and Xue-jun Li have nothing to disclose.

Compliance with Ethics Guidelines. This work was approved by the Medical Research and Ethics Committee of the First Affiliated Hospital, Xiamen University (Xiamen, China). This work was registered at International Clinical Trials Registry Platform (ICTRP) with trial registration identifier number NCT03563794.
Informed consent was obtained from all participants in this study.

Open Access. This article is distributed under the terms of the Creative Commons Attribution-NonCommercial 4.0 International License (http://creativecommons.org/licenses/ by-nc/4.0/), which permits any noncommercial use, distribution, and reproduction in any medium, provided you give appropriate credit to the original author(s) and the source, provide a link to the Creative Commons license, and indicate if changes were made.

\section{REFERENCES}

1. Kramer CK, Zinman B, Retnakaran R. Short-term intensive insulin therapy in type 2 diabetes mellitus: a systematic review and meta-analysis. Lancet Diabetes Endocrinol. 2013;1(1):28-34.

2. Weng J, Li Y, Xu W, et al. Effect of intensive insulin therapy on beta-cell function and glycaemic control in patients with newly diagnosed type 2 diabetes: a multicentre randomised parallel-group trial. Lancet. 2008;371(9626):1753-60.

3. Lv WS, Li L, Wen JP, et al. Comparison of a multiple daily insulin injection regimen (glargine or detemir once daily plus prandial insulin aspart) and continuous subcutaneous insulin infusion (aspart) in short-term intensive insulin therapy for poorly controlled type 2 diabetes patients. Int J Endocrinol. 2013;2013:614242.

4. Yang $\mathrm{H}$, Heng $\mathrm{X}$, Liang $\mathrm{C}$, et al. Comparison of continuous subcutaneous insulin infusion and multiple daily insulin injections in Chinese patients with type 2 diabetes mellitus. J Int Med Res. 2014;42(4):1002-10.

5. Pastores SM. ACP Journal Club. Review: Intensive insulin therapy does not reduce mortality but increases severe hypoglycemia in hospitalized patients. Ann Intern Med. 2011;155(2):JC1-12.

6. Nyenwe E. Intensive insulin therapy in hospitalised patients increases the risk of hypoglycaemia and has no effect on mortality, infection risk or length of stay. Evid Based Med. 2012;17(1):8-9.

7. Brownlee M. Biochemistry and molecular cell biology of diabetic complications. Nature. 2001;414(6865):813-20. 
8. Brownlee M. The pathobiology of diabetic complications: a unifying mechanism. Diabetes. 2005;54(6):1615-25.

9. Johnson EL. Glycemic variability in type 2 diabetes mellitus: oxidative stress and macrovascular complications. Adv Exp Med Biol. 2012;771:139-54.

10. Piconi L, Quagliaro L, Assaloni R, et al. Constant and intermittent high glucose enhances endothelial cell apoptosis through mitochondrial superoxide overproduction. Diabetes Metab Res Rev. 2006;22(3):198-203.

11. Ceriello A, Esposito K, Piconi L, et al. Oscillating glucose is more deleterious to endothelial function and oxidative stress than mean glucose in normal and type 2 diabetic patients. Diabetes. 2008;57(5): 1349-54.

12. Zhao Y, Yang L, Zhou Z. Dipeptidyl peptidase-4 inhibitors: multitarget drugs, not only antidiabetes drugs. J Diabetes. 2014;6(1):21-9.

13. Ji LN, Pan CY, Lu JM, et al. Efficacy and safety of combination therapy with vildagliptin and metformin versus metformin uptitration in Chinese patients with type 2 diabetes inadequately controlled with metformin monotherapy: a randomized, open-label, prospective study (VISION). Diabetes Obes Metab. 2016;18(8):775-82.

14. Strain WD, Lukashevich V, Kothny W, Hoellinger MJ, Paldanius PM. Individualised treatment targets for elderly patients with type 2 diabetes using vildagliptin add-on or lone therapy (INTERVAL): a 24 week, randomised, double-blind, placebo-controlled study. Lancet. 2013;382(9890):409-16.

15. Ahren B, Mathieu C, Bader G, Schweizer A, Foley JE. Efficacy of vildagliptin versus sulfonylureas as addon therapy to metformin: comparison of results from randomised controlled and observational studies. Diabetologia. 2014;57(7):1304-7.

16. Lukashevich V, Del Prato S, Araga M, Kothny W. Efficacy and safety of vildagliptin in patients with type 2 diabetes mellitus inadequately controlled with dual combination of metformin and sulphonylurea. Diabetes Obes Metab. 2014;16(5):403-9.

17. Odawara M, Hamada I, Suzuki M. Efficacy and safety of vildagliptin as add-on to metformin in
Japanese patients with type 2 diabetes mellitus. Diabetes Ther. 2014;5(1):169-81.

18. Hare KJ, Vilsboll T, Asmar M, Deacon CF, Knop FK, Holst JJ. The glucagonostatic and insulinotropic effects of glucagon-like peptide 1 contribute equally to its glucose-lowering action. Diabetes. 2010;59(7): 1765-70.

19. Nalysnyk L, Hernandez-Medina M, Krishnarajah G. Glycaemic variability and complications in patients with diabetes mellitus: evidence from a systematic review of the literature. Diabetes Obes Metab. 2010;12(4):288-98.

20. Rizzo MR, Barbieri M, Marfella R, Paolisso G. Reduction of oxidative stress and inflammation by blunting daily acute glucose fluctuations in patients with type 2 diabetes: role of dipeptidyl peptidase-IV inhibition. Diabetes Care. 2012;35(10):2076-82.

21. Farngren J, Persson M, Schweizer A, Foley JE, Ahren B. Glucagon dynamics during hypoglycaemia and food-re-challenge following treatment with vildagliptin in insulin-treated patients with type 2 diabetes. Diabetes Obes Metab. 2014;16(9):812-8.

22. Pi-Sunyer FX. The effects of pharmacologic agents for type 2 diabetes mellitus on body weight. Postgrad Med. 2008;120(2):5-17.

23. Panina G. The DPP-4 inhibitor vildagliptin: robust glycaemic control in type 2 diabetes and beyond. Diabetes Obes Metab. 2007;9[Suppl 1]:32-9.

24. Kanazawa I, Tanaka KI, Notsu M, et al. Long-term efficacy and safety of vildagliptin add-on therapy in type 2 diabetes mellitus with insulin treatment. Diabetes Res Clin Pract. 2017;123:9-17.

25. Kothny W, Foley J, Kozlovski P, Shao Q, Gallwitz B, Lukashevich V. Improved glycaemic control with vildagliptin added to insulin, with or without metformin, in patients with type 2 diabetes mellitus. Diabetes Obes Metab. 2013;15(3):252-7.

26. Ning G, Wang W, Li L, et al. Vildagliptin as add-on therapy to insulin improves glycemic control without increasing risk of hypoglycemia in Asian, predominantly Chinese, patients with type 2 diabetes mellitus. J Diabetes. 2016;8(3):345-53. 